\title{
Guided Internet-Based Cognitive Behavioral Therapy in Japanese Patients With Obsessive-Compulsive Disorder: Protocol for a Randomized Controlled Trial
}

Kazuki Matsumoto ${ }^{1}$, PhD; Sayo Hamatani ${ }^{1,2}$, PhD; Takuya Makino ${ }^{3,4}$; Taku Uemura ${ }^{3}$; Futoshi Suzuki ${ }^{3,4}, \mathrm{MD}, \mathrm{PhD}$; Seina Shinno, ${ }^{5,6}$;omoki Ikai ${ }^{7}, \mathrm{MD}, \mathrm{PhD}$; Hiroyuki Hayashi ${ }^{6,8}, \mathrm{MD}, \mathrm{PhD}$; Chihiro Sutoh${ }^{9}, \mathrm{MD}, \mathrm{PhD}$; Eiji Shimizu ${ }^{1,9}$, $\mathrm{MD}, \mathrm{PhD}$

\footnotetext{
${ }^{1}$ Research Center for Child Mental Development, Chiba University, Chiba, Japan

${ }^{2}$ Japan Society for the Promotion of Science, Tokyo, Japan

${ }^{3}$ Research Center for Child Mental Development, University of Fukui, Fukui, Japan

${ }^{4}$ Department of Child and Adolescent Psychological Medicine, University of Fukui Hospital, Fukui, Japan

${ }^{5}$ Department of Integrated Medical Sciences, Graduate School of Medicine, University of Fukui, Fukui, Japan

${ }^{6}$ Department of Integrated Advanced Medicine, Graduate School of Medicine, University of Fukui, Fukui, Japan

${ }^{7}$ Department of Primary Health Care, Faculty of Medicine, University of Fukui, Fukui, Japan

${ }^{8}$ Department of Emaergency and General Medicine, University of Fukui Hospital, Fukui, Japan

${ }^{9}$ Department of Cognitive Behavioral Physiology, Graduate School of Medicine, Chiba University, Chiba, Japan
}

\section{Corresponding Author:}

Kazuki Matsumoto, PhD

Research Center for Child Mental Development

Chiba University

1-8-1, Inohana

Chuo-ku

Chiba, 2608670

Japan

Phone: 81080432262975

Email: axpa0219@chiba-u.jp

\section{Abstract}

Background: Cognitive behavioral therapy for obsessive-compulsive disorder has been established, but access to this therapy in Japan is limited. Internet-based cognitive behavioral therapy may improve treatment accessibility and sufficiently improve obsessive-compulsive symptoms. There are few randomized controlled trials examining the effectiveness of internet-based cognitive behavioral therapy in patients with obsessive-compulsive disorder. We designed a randomized controlled trial protocol to assess the effectiveness of guided internet-based cognitive behavioral therapy in Japanese patients with obsessive-compulsive disorder.

Objective: We aimed to develop a protocol for a randomized controlled trial of internet-based cognitive behavioral therapy in Japanese patients with obsessive-compulsive disorder.

Methods: The randomized controlled trial will compare internet-based cognitive behavioral therapy treatment and usual care groups, each consisting of 15 participants $(n=30)$ diagnosed with obsessive-compulsive disorder. We will evaluate the effectiveness of a 12-week intervention. The primary outcome of symptom severity will be measured using the Yale-Brown Obsessive-Compulsive Scale. Secondary outcomes will be assessed with the Obsessive-Compulsive Inventory, Beck Anxiety Inventory, Patient Health Questionnaire-9, Generalized Anxiety Disorder-7, Working Alliance Inventory-Short Form, and the Euro Qol - 5 Dimension. All measures will be assessed at weeks 0 (baseline) and 12 (follow-up). In the statistical analysis comparing treatment effects, the least-squares means and their 95\% CIs will be estimated by analysis of covariance with the change in total outcomes scores at week 12. All comparisons are planned, and all $P$ values will be two-sided, with values <.05 considered statistically significant.

Results: The study will be performed from January 2020 to March 2021, and results are expected to be available in mid-2021. 
Conclusions: The trial will demonstrate whether internet-based cognitive behavioral therapy improves access and is more effective than more usual care for patients with obsessive-compulsive disorder in Japan.

Trial Registration: University Hospital Medical Information Network (UMIN) 000039375; https://upload.umin.ac.jp/cgi-open-bin/ctr/ctr_view.cgi?recptno=R000044422

International Registered Report Identifier (IRRID)： DERR1-10.2196/18216

(JMIR Res Protoc 2020;9(6):e18216) doi: 10.2196/18216

\section{KEYWORDS}

internet-based cognitive behavioral therapy; cognitive behavioral therapy; obsessive-compulsive disorder; randomized controlled trial; protocol

\section{Introduction}

\section{Background}

Obsessive-compulsive disorder (OCD) has been defined as a common, chronic, and long-lasting disorder in which a person has uncontrollable, reoccurring thoughts (obsessions) and/or behaviors (compulsions) that he or she feels the urge to repeat [1]. OCD is characterized by uncomfortable and painful obsessions and repeated obsessions. The 12-month prevalence of anxiety disorder including OCD in the Japanese adult general population is $5.3 \%$, making it the most common psychiatric disorder [2]. Systemic reviews and meta-analysis show that cognitive behavioral therapy (CBT) is the most effective treatment for OCD [3] and is recommended as first-line therapy by the treatment guidelines of The National Institute for Health and Care Excellence in the United Kingdom (UK NICE) [4]. Telemedicine or remote treatment for patients living in rural areas via internet-based cognitive behavioral therapy (ICBT) has been established as a standard of care in Stockholm, Sweden [5]. In ICBT, patients and therapists interact primarily via email, with treatment including routine work such as explaining the symptoms of the patient's condition and introducing coping skills based on cognitive behavioral science. A systematic review with meta-analysis comparing 21 studies of face-to-face and guided self-help CBT (mostly ICBT) in 810 patients showed no clear differences in their treatment effect: The effect size was Cohen $d=-0.02$, and face-to-face CBT was smaller than ICBT [6]. Unlike ICBT for anxiety and depression, effectiveness of ICBT for OCD has been investigated in clinical trials. Japanese patients with OCD have significantly improved symptoms with ICBT via videoconference and the method achieved high acceptance [7,8]. Prior study results suggest guided ICBT with minimal therapist intervention may be as effective and accepted by Japanese patients with OCD as ICBT via videoconference [9].

\section{Internet-Based Cognitive Behavioral Therapy for Obsessive-Compulsive Disorder}

Previous randomized controlled trials for obsessive-compulsive disorder have been performed in Sweden, the United States, and Korea [10-12]. In a previous randomized study of 101 patients with OCD assigned to ICBT and online supportive psychotherapy followed by blinded assessments [10], ICBT showed significant improvements in the intervention group compared to the control group. Obsessive-compulsive symptoms as measured by the Yale-Brown Obsessive-Compulsive Scale (Y-BOCS) were improved, and the effect size was high (Cohen $d=1.12$ ). Another study of 56 patients with OCD included ICBT, reading therapy, and waiting groups [11]. The results of that study suggested that ICBT has a large effect size (Cohen $d=1.57$ ) among the waiting group at pre- and post- treatment. In Asia, a Korean research team conducted a randomized controlled trial and reported that the treatment group showed significantly improved symptoms over the waiting group, and 25.9\% (17/42) responded to treatment, with a within-group effect size of 1.64 (Cohen $d$ ) [12]. Thus, ICBT, like face-to-face CBT [13], has been shown to be highly effective in the treatment of OCD.

More clinical trials are needed to draw general conclusions regarding the efficacy of ICBT because of substantial country-to-country variations in cultural background, the spread of information, availability of communication equipment, and literacy. Prior research has been performed in countries where the environment is conducive to ICBT, especially as developed countries have a well-established computerized social infrastructure [14]. The penetration rate of information and communication equipment in Japan is more than $90 \%$ of all households [15], and there is a favorable social infrastructure for verifying the effectiveness of ICBT. In Japan, our research team confirmed that all patients were in remission by conducting CBT on 3 patients with OCD in their 20s and 40s in the ICBT case series [9]. Based on this achievement, we developed an ICBT program that includes an electronic learning (e-learning) system and chat app. From January 2020 to March 2021, we are conducting a randomized controlled clinical trial in patients diagnosed with OCD.

\section{Objective}

This paper describes the study protocol for a randomized controlled trial designed to evaluate the clinical effectiveness of ICBT versus usual care (UC) among patients diagnosed with OCD.

\section{Methods}

\section{Study design}

This study was designed as a prospective blinded randomized trial with two parallel intervention groups consisting of a 12-week treatment regime of UC alone or ICBT combined with UC (Figure 1) [15-18]. 
Figure 1. CONSORT flow diagram of a parallel randomized trial with two groups.

\section{Enrollment}

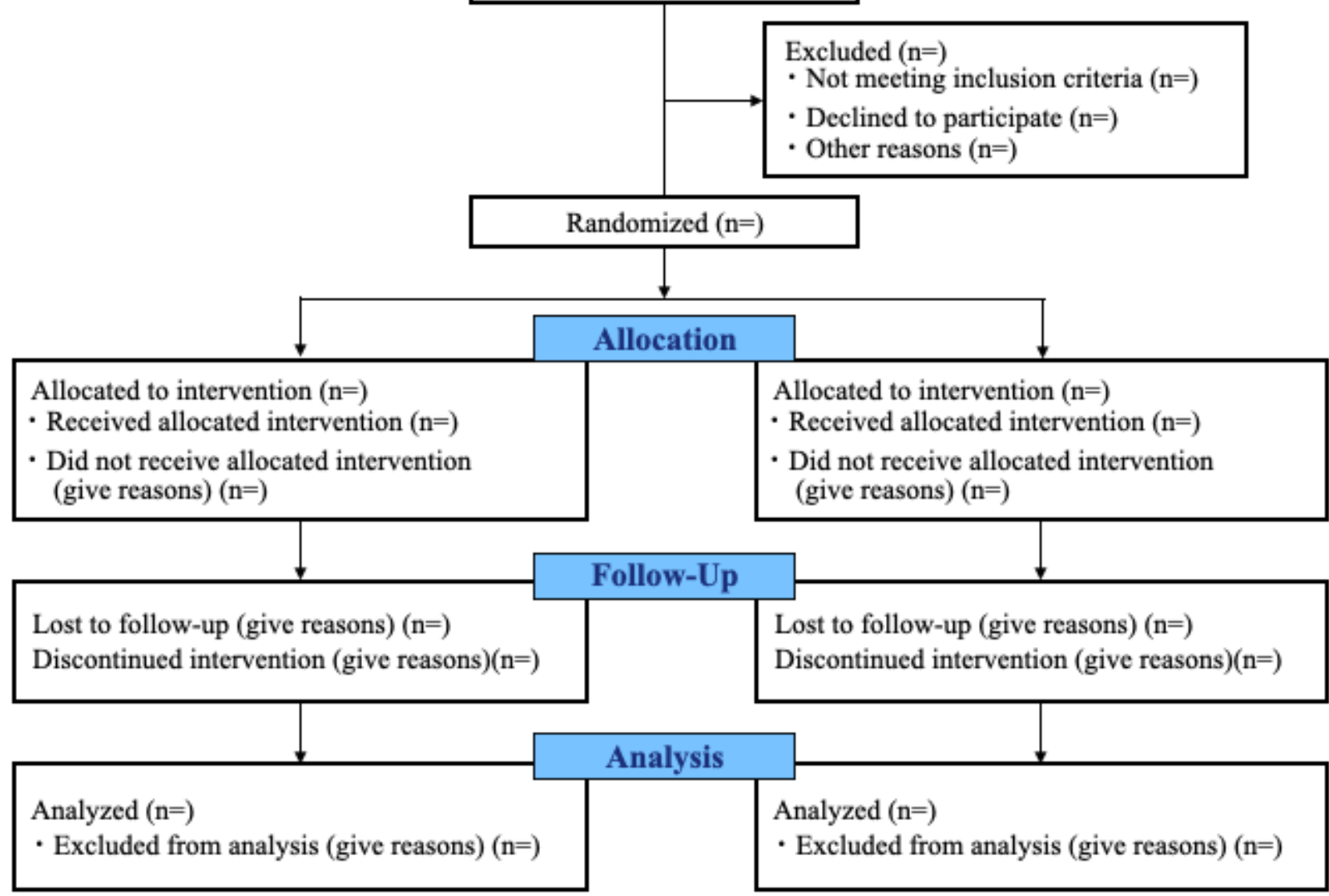

\section{Participants and Eligibility Criteria}

Inclusion criteria for this study include individuals aged 15-60 years having a primary diagnosis of OCD according to the Mini-International Neuropsychiatric Interview (MINI) and remaining symptomatic $[19,20]$ with symptoms rated at least moderate in severity, based on a Y-BOCS score >14 [21,22], and with sufficient skills to send email and access the e-learning system. Participants with psychosis or organic mental disorder, or with a current high risk of suicide, substance abuse or dependence within the 12 months prior to enrollment, antisocial personality disorder, or unstable medical conditions will be excluded.

\section{Recruitment}

The planned recruitment rate is 3 participants per month from January 2020 to December 2020, or until a total of 30 participants are recruited. Participants will be recruited by placing informational posters and leaflets at medical institutions in Chiba and Fukui Prefecture and on the institutional homepage. All participants will continue to be treated by their general practitioners, whose permission must be granted prior to study enrollment. This study will be conducted at the outpatient clinic of Chiba University Hospital and Fukui University Hospital in Japan.

\section{Interventions}

\section{Guided ICBT program}

The ICBT program participants will engage in one program learning session per week. After working on the ICBT, the patient will email the therapist about their thoughts and questions about the study and behaviors suggested by the program for them to try in daily life. The therapist, as general rule, will contact the patient within 24 hours to encourage the patient's efforts, raise questions, and advise on CBT techniques to increase the effectiveness of treatment. The ICBT program consists of 12 weekly lessons, including the following elements: ICBT program guidance, psychological education and case conceptualization of obsessive-compulsive disorder, setting treatment goals and creating an anxiety hierarchy, explaining the behavioral experiment of catastrophic interpretation and the exposure-response disturbance method, and preventing recurrence.

\section{Software}

We will use LearningBox, a system developed by Tatsuno Information Systems [23] and MediLine, a medical chat service developed by Shar Medical to provide ICBT [24]. LearningBox (Multimedia Appendix 1) is an e-learning system that allows administrators to easily create and manage educational materials, manage members, and save and view grades. Video and PDF teaching materials can also be posted on the site and distributed to specific users. The e-learning system can store and manage 
user results, but personal information is not stored during the present trial.

MediLine (Multimedia Appendix 2) is a medical chat service (medical social networking service) that replaces email and phone calls. It has strong encryption against military-level information leaks. MediLine communicates in a double-encrypted state according to the Japanese government guidelines [25-28]. Encryption is performed end-to-end in real time in the server, during communication, and in temporary memory at the terminal. In other words, it is designed so that information cannot be extracted during data transfer. Since identifiers are issued by in-house introduction, hijacking and spoofing are prevented. The staff registered by the organization can delete the account at the time of retirement and cannot view it thereafter, so there is no provision for information to be taken out of the hospital after retirement [24].

\section{Usual Care (Control group)}

Participants will be permitted to continue using antidepressant or other medicines during the study period. Participants' primary doctor will have right to change medication, to refer participants for counselling, and to secondary care if deemed clinically appropriate. All changes in conventional treatment, along with the reasons for those changes, will be recorded. Participants in the control group will be offered the ICBT treatment after the trial if they did not improve under the control conditions.

\section{Outcomes}

\section{Baseline and Clinical Characteristics}

Baseline characteristics will include gender, age, educational attainment, marital status, employment status, age at onset and duration of OCD, medications, and intelligence as measured by the Japanese Adult Rating Test (JART) [29,30].

\section{Primary Outcome}

The primary outcome will be measured by the Y-BOCS, which is a rated questionnaire consisting of 10 questions across two subscales, Obsession and Compulsion [21,22].

\section{Secondary Outcomes}

Secondary outcomes will include health-related quality of life, symptoms of depression and generalized anxiety, and therapeutic relationship. We will measure health-related quality of life with the EuroQol - 5 Dimension (EQ-5D) [31,32], the psychological bond between therapist and participant using the Working Alliance Inventory-Short Form (WAI-SF) [33], depressive symptoms using the Patient Health Questionnaire-9 (PHQ-9) [34], generalized anxiety symptoms using the Generalized Anxiety Disorder-7 (GAD-7) [35], and the Beck Anxiety Inventory (BAI) [36].

\section{Sample Size}

We used the statistical analysis software $G^{*}$ power 3.1 to estimate sample size of an unmatched t-test [37]. Sample size was estimated as 14 participants per group. The effect size predicted in this study is at least 1.00 from the two previous two clinical trials [8-10], the directionality of the test is a two-sided test, the significance level was set at $0.05 \%$, the test was two-sided, and the test power (1- $\beta$ ) was set to $80 \%$. The study will require a minimum 14 participants per group so we are setting a target enrollment of 30 participants to allow for a $5 \%$ dropout rate.

\section{Randomization}

Eligible participants will be randomly assigned to the ICBT or UC group at a ratio of $1: 1$, with assignments made using the minimization method, ensuring a balance in baseline Y-BOCS scores (Y-BOCS $\geq 20$ ) and gender.

\section{Statistical Analysis Plan}

Statistical analysis and reporting will be conducted in accordance with CONSORT (Consolidated Standards of Reporting Trials) guidelines [38], with primary analyses based on the intention-to-treat principle. For baseline variables, summary statistics will be constructed, employing frequencies and proportions for categorical data and mean and SD for continuous variables. Baseline variables will be compared using the Fisher exact test for categorical outcomes and the unpaired $t$-test for continuous variables. For the primary analysis comparing treatment effects, the least-squares means and their 95\% CIs will be estimated by analysis of covariance (ANCOVA) with the change in total Y-BOCS scores at week 12. This ANCOVA model will account for the variation caused by treatment effects, and gender and baseline Y-BOCS scores will be entered as covariates. Analyses of secondary outcomes will be performed in the same manner. All comparisons are planned, and all $P$ values will be two-sided. $P$ values $<.05$ will be considered statistically significant. All statistical analyses will be performed using the latest version of SAS (SAS Institute Inc).

\section{Ethics and Dissemination}

This study will be conducted at the Academic Outpatient Clinics of Chiba University and Fukui University in Japan. When potential participants contact the study trial office through the Chiba University, they will be informed of the study objectives and be asked if they are willing to participate by phone screening. Each participant will then be required to provide written informed consent for their participation in this study. Each participant will be informed that all participants will receive UC from their general practitioner and that half of the recruited participants will also receive ICBT in addition to their UC. All adverse events will be reported, and serious adverse events will be immediately reported to the Institutional Review Board of Chiba University Hospital in addition to being registered with the hospital risk management system. An adverse event will be defined as a symptom or disease occurring during the clinical trial, whether related to the ICBT program or not. The study will be conducted and reported according to CONSORT-ETHICS guideline recommendations [39].

This clinical trial protocol was approved by the clinical trial review committee of Chiba University Hospital on November 18, 2019 (G2019017) and approved by the ethics review committee of the Fukui University Graduate School (20190075). It is registered in the University Hospital Medical Information Network database of clinical trials in Japan (UMIN: 000044422). 


\section{Results}

The study period is from January 6, 2020 to March 31, 2021. The case registration period is scheduled for 12 months from January 6, 2020 to December 31, 2020.

\section{Discussion}

This randomized controlled study will evaluate the effectiveness of ICBT for patients with OCD. The findings of this study will provide valuable evidence to facilitate development and establish treatment algorithms in ICBT for patients with OCD. The study will also introduce this method for providing ICBT in Japan, as patients will be given access to self-help materials using an e-learning system (LearningBox) and medical chat app (MediLine). The UC control group will be randomly assigned, and the multicenter study design will reduce bias and improve the likelihood of obtaining generalizable results. The study will also have limitations, including the inability to elucidate the specific effects of ICBT program because there is no placebo group to control for nonspecific factors. The study also will not control for medical therapies or treatment resistance. It is unclear whether pharmacotherapy plus CBT improves OCD symptoms more than pharmacotherapy alone [4,40]. Therefore, combination therapy with pharmaceuticals and ICBT should be tested in future trials to determine whether each is beneficial or if therapy is improved by combined therapies.

\section{Acknowledgments}

This study was supported by the Japan Society for the Promotion of Science KAKENHI Grant-in-Aid for Scientific Research, Grant Number 18K03130 and 18K17313.

\section{Conflicts of Interest}

None declared.

\section{Multimedia Appendix 1}

LearningBox E-Learning System.

[PNG File, 219 KB-Multimedia Appendix 1]

\section{Multimedia Appendix 2}

The MediLine Chat App.

[PNG File, 139 KB-Multimedia Appendix 2]

\section{References}

1. American PA. Diagnostic Statistical Manual of Mental Disorders, Fifth Edition. Arlington, VA. United States: American Psychiatric Publishing; 2013:A.

2. Kawakami N, Takeshima T, Ono Y, Uda H, Hata Y, Nakane Y, et al. Twelve-month prevalence, severity, and treatment of common mental disorders in communities in Japan: preliminary finding from the World Mental Health Japan Survey 2002-2003. Psychiatry Clin Neurosci 2005 Aug;59(4):441-452. [doi: 10.1111/j.1440-1819.2005.01397.x] [Medline: $\underline{16048450]}$

3. Hofmann SG, Smits JAJ. Cognitive-behavioral therapy for adult anxiety disorders: a meta-analysis of randomized placebo-controlled trials. J Clin Psychiatry 2008 Apr;69(4):621-632 [FREE Full text] [doi: 10.4088/jcp.v69n0415] [Medline: $\underline{18363421]}$

4. National IFHE(. Clinical guideline CG31. 2005 Nov. Obsessive-compulsive disorder and body dysmorphic: disorder treatment URL: https://www.nice.org.uk/guidance/CG31/chapter/ 1-Guidance\#steps-35-treatment-options-for-people-with-ocd-or-bdd [accessed 2020-05-12]

5. Andersson G. The Internet and CBT: A Clinical Guide. In: CRC Press, a member of the Taylor \& Group. 5 Howick Place, London, SW1P 1WG: CRC Press, a member of the Taylor \& Group; 2015:A.

6. Cuijpers P, Marks IM, van Straten A, Cavanagh K, Gega L, Andersson G. Computer-aided psychotherapy for anxiety disorders: a meta-analytic review. Cogn Behav Ther 2009;38(2):66-82. [doi: 10.1080/16506070802694776] [Medline: 20183688]

7. Matsumoto K, Sutoh C, Asano K, Seki Y, Urao Y, Yokoo M, et al. Internet-Based Cognitive Behavioral Therapy With Real-Time Therapist Support via Videoconference for Patients With Obsessive-Compulsive Disorder, Panic Disorder, and Social Anxiety Disorder: Pilot Single-Arm Trial. J Med Internet Res 2018 Dec 17;20(12):e12091-e12890 [FREE Full text] [doi: 10.2196/12091] [Medline: 30559094]

8. Matsumoto K, Yoshida T, Hamatani S, Sutoh C, Hirano Y, Shimizu E. Prognosis Prediction Using Therapeutic Agreement of Video Conference-Delivered Cognitive Behavioral Therapy: Retrospective Secondary Analysis of a Single-Arm Pilot Trial. JMIR Ment Health 2019 Nov 15;6(11):e15747 [FREE Full text] [doi: 10.2196/15747] [Medline: 31730037] 
9. Matsumoto K, Hamatani S, Shimizu E, Yoshino K, Shirayama Y, Sato K. Digital Teaching Materials of Cognitive Behavioral Therapy for Obsessive-Compulsive Disorder: Case Series. Japanese Society of Anxiety and Related Disorders, in-press 2020 Sep (forthcoming).

10. Andersson E, Enander J, Andrén P, Hedman E, Ljótsson B, Hursti T, et al. Internet-based cognitive behaviour therapy for obsessive-compulsive disorder: a randomized controlled trial. Psychol. Med 2012 Feb 21;42(10):2193-2203. [doi: 10.1017/s0033291712000244]

11. Wootton B, Dear B, Johnston L, Terides M, Titov N. Remote treatment of obsessive-compulsive disorder: A randomized controlled trial. Journal of Obsessive-Compulsive and Related Disorders 2013 Oct;2(4):375-384 [FREE Full text] [doi: 10.1016/j.jocrd.2013.07.002]

12. Seol S, Kwon JS, Kim YY, Kim SN, Shin M. Internet-Based Cognitive Behavioral Therapy for Obsessive-Compulsive Disorder in Korea. Psychiatry Investig 2016 Jul;13(4):373-382 [FREE Full text] [doi: 10.4306/pi.2016.13.4.373] [Medline: 27482237]

13. Carlbring P, Andersson G, Cuijpers P, Riper H, Hedman-Lagerlöf E. Internet-based vs. face-to-face cognitive behavior therapy for psychiatric and somatic disorders: an updated systematic review and meta-analysis. Cogn Behav Ther 2018 Jan;47(1):1-18. [doi: 10.1080/16506073.2017.1401115] [Medline: 29215315]

14. Miniwatts MG. Internet Growth Statistics. Internet World Stats URL: https://www.internetworldstats.com/emarketing.htm [accessed 2020-05-12]

15. Ministry of Internal Affairs and Communications. WHITE PAPER Information and Communications in Japan 2018 URL: http://www.soumu.go.jp/johotsusintokei/whitepaper/h30.html [accessed 2020-05-12]

16. Moher D, Schulz KF, Altman DG, CONSORT GROUP (Consolidated Standards of Reporting Trials). The CONSORT statement: revised recommendations for improving the quality of reports of parallel-group randomized trials. Ann Intern Med 2001 Apr 17;134(8):657-662. [doi: 10.7326/0003-4819-134-8-200104170-00011] [Medline: 11304106]

17. Moher D, Schulz KF, Altman D. The CONSORT statement: revised recommendations for improving the quality of reports of parallel-group randomized trials. JAMA 2001 Apr 18;285(15):1987-1991. [Medline: 11308435]

18. Moher D, Schulz KF, Altman DG. The CONSORT statement: revised recommendations for improving the quality of reports of parallel-group randomised trials. Lancet 2001 Apr 14;357(9263):1191-1194. [Medline: 11323066]

19. Sheehan DV, Lecrubier Y, Sheehan KH, Amorim P, Janavs J, Weiller E, et al. The Mini-International Neuropsychiatric Interview (M.I.N.I.): the development and validation of a structured diagnostic psychiatric interview for DSM-IV and ICD-10. J Clin Psychiatry 1998;59 Suppl 20:22-33;quiz 34. [Medline: 9881538]

20. Muramatsu K, Miyaoka H, Kamijima K, Muramatsu Y, Yoshida M, Otsubo T, et al. The patient health questionnaire, Japanese version: validity according to the mini-international neuropsychiatric interview-plus. Psychol Rep 2007 Dec;101(3 Pt 1):952-960. [doi: 10.2466/pr0.101.3.952-960] [Medline: 18232454]

21. Goodman WK, Price LH, Rasmussen SA, Mazure C, Fleischmann RL, Hill CL, et al. The Yale-Brown Obsessive Compulsive Scale. I. Development, use, and reliability. Arch Gen Psychiatry 1989 Nov;46(11):1006-1011. [Medline: 2684084]

22. Hamagaki S, Takagi S, Urushihara Y, Ishisaka Y, Matsumoto M. Development and use of the Japanese version of the self-report Yale-Brown Obsessive Compulsive Scale. Seishin Shinkeigaku Zasshi 1999;101(2):152-168. [Medline: 10375975]

23. Tatsuno Information System Co. LearningBox ${ }^{\circledR}$ URL: https://lms.quizgenerator.net [accessed 2020-05-12]

24. Shar Medical Co. MediLine® URL: https://www.mediline.jp [accessed 2020-05-12]

25. Ministry of Health, Labour and Welfare. 2017. The guideline for safety management of medical information systems, 5th edition URL: https://www.mhlw.go.jp/file/05-Shingikai-12601000-Seisakutoukatsukan-Sanjikanshitsu_Shakaihoshoutantou/ 0000166260.pdf [accessed 2020-05-12]

26. Ministry OIA. Providers handle medical information, version 1 . The guideline for safety management when ASP/SaaS URL: http://www.soumu.go.jp/menu_news/s-news/01ryutsu02_01000009.html [accessed 2020-05-12]

27. Ministry of Economy, Trade and Industry. 2008. The guideline of information security countermeasure in ASP/Saas URL: https://www.soumu.go.jp/ict seisan/ [accessed 2020-05-12]

28. Ministry of Economy, Trade and Industry. 2012. The guideline for information processing companies that consign and manage medical information, 2nd edition URL: https://www.meti.go.jp/policy/it policy/privacy/iryouglv2.pdf [accessed 2020-05-12]

29. Matsuoka K, Uno M, Kasai K, Koyama K, Kim Y. Estimation of premorbid IQ in individuals with Alzheimer's disease using Japanese ideographic script (Kanji) compound words: Japanese version of National Adult Reading Test. Psychiatry Clin Neurosci 2006 Jun;60(3):332-339 [FREE Full text] [doi: 10.1111/j.1440-1819.2006.01510.x] [Medline: 16732750]

30. Nelson H, Willison J. The revised national adult reading test - Test manual. Windsor, UK: NFER-Nelson; 1991.

31. EuroQol G. EuroQol--a new facility for the measurement of health-related quality of life. Health Policy 1990 Dec;16(3):199-208. [Medline: 10109801]

32. Tsuchiya A, Ikeda S, Ikegami N, Nishimura S, Sakai I, Fukuda T, et al. Estimating an EQ-5D population value set: the case of Japan. Health Econ 2002 Jun;11(4):341-353. [doi: 10.1002/hec.673] [Medline: 12007165]

33. Tracey TJ, Kokotovic AM. Factor structure of the Working Alliance Inventory. Psychological Assessment: A Journal of Consulting and Clinical Psychology 1989 Sep;1(3):207-210. [doi: 10.1037/1040-3590.1.3.207] 
34. Spitzer RL, Kroenke K, Williams JB. Validation and utility of a self-report version of PRIME-MD: the PHQ primary care study. Primary Care Evaluation of Mental Disorders. Patient Health Questionnaire. JAMA 1999 Nov 10;282(18):1737-1744. [Medline: 10568646$]$

35. Spitzer RL, Kroenke K, Williams JBW, Löwe B. A brief measure for assessing generalized anxiety disorder: the GAD-7. Arch Intern Med 2006 May 22;166(10):1092-1097. [doi: 10.1001/archinte.166.10.1092] [Medline: 16717171]

36. Beck AT, Epstein N, Brown G, Steer RA. An inventory for measuring clinical anxiety: psychometric properties. J Consult Clin Psychol 1988 Dec;56(6):893-897. [Medline: 3204199]

37. Heine H. G*Power: Statistical Power Analyses for Windows and Mac. G*Power: Statistical Power Analyses for Windows and Mac URL: https://www.psychologie.hhu.de/arbeitsgruppen/allgemeine-psychologie-und-arbeitspsychologie/gpower. html [accessed 2020-05-12]

38. Schulz KF, Altman DG, Moher D. CONSORT 2010 Statement: updated guidelines for reporting parallel group randomised trials. Trials 2010 Mar 24;11(1) [FREE Full text] [doi: 10.1186/1745-6215-11-32]

39. Eysenbach G, CONSORT-EHEALTH Group. CONSORT-EHEALTH: improving and standardizing evaluation reports of Web-based and mobile health interventions. J Med Internet Res 2011 Dec 31;13(4):e126 [FREE Full text] [doi: 10.2196/jmir.1923] [Medline: 22209829]

40. Hofmann SG, Sawyer AT, Korte KJ, Smits JAJ. Is it Beneficial to Add Pharmacotherapy to Cognitive-Behavioral Therapy when Treating Anxiety Disorders? A Meta-Analytic Review. Int J Cogn Ther 2009 Jan 01;2(2):160-175. [doi: 10.1521/ijct.2009.2.2.160] [Medline: 19714228]

\author{
Abbreviations \\ BAI: Beak Anxiety Inventory \\ CBT: Cognitive Behavioral Therapy \\ EQ-5D: EuroQol-5-Divison \\ GAD-7: Generalized Anxiety Disorder-7 \\ ICBT: Internet-Based Cognitive Behavioral Therapy \\ JART: Japanese Adult Rating Test \\ OCD: Obsessive-Compulsive Disorder \\ PHQ-9: Patient Health Questionnaire-9 \\ RCT: Randomized Controlled Trial \\ UC: Usual care \\ WAI-SF: Working Alliance Inventory-Short From \\ Y-BOCS: Yale-Brown Obsessive-Compulsive Scale
}

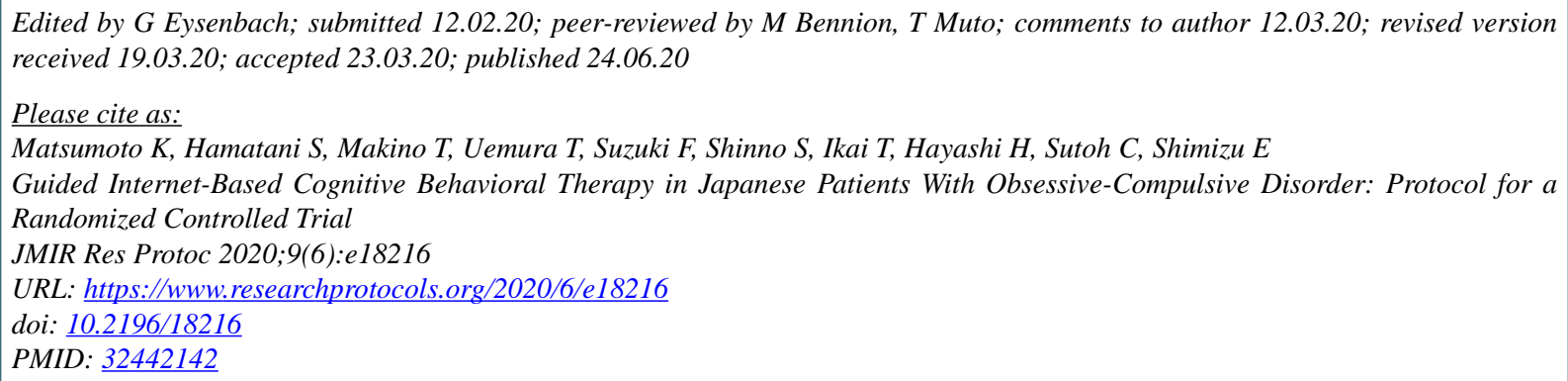

CKazuki Matsumoto, Sayo Hamatani, Takuya Makino, Taku Uemura, Futoshi Suzuki, Seina Shinno, Tomoki Ikai, Hiroyuki Hayashi, Chihiro Sutoh, Eiji Shimizu. Originally published in JMIR Research Protocols (http://www.researchprotocols.org), 24.06.2020. This is an open-access article distributed under the terms of the Creative Commons Attribution License (https://creativecommons.org/licenses/by/4.0/), which permits unrestricted use, distribution, and reproduction in any medium, provided the original work, first published in JMIR Research Protocols, is properly cited. The complete bibliographic information, a link to the original publication on http://www.researchprotocols.org, as well as this copyright and license information must be included. 